\title{
MR-guided focused ultrasound for the treatment of adenomyosis: current insights
}

This article was published in the following Dove Press journal:

Research and Reports in Focused Ultrasound

24 December 2015

Number of times this article has been viewed

\section{Wenzhuang Chin Jocelyn R Walbridge Stephen D Quinn}

Department of Obstetrics and Gynaecology, St Mary's Hospital, Imperial College Healthcare NHS Trust, London, UK
Correspondence: Stephen D Quinn Department of Obstetrics and Gynaecology, Mint Wing, St Mary's Hospital, South Wharf Road, London W2 INY, UK

Tel +44203312 III7

Email s.quinn@imperial.ac.uk

\begin{abstract}
Adenomyosis is a benign gynecological condition causing significant morbidity among women of reproductive age. The available treatments are currently limited in number and efficacy. While hysterectomy is curative, it is unacceptable to many women, particularly those wishing to conserve their fertility. Magnetic resonance-guided focused ultrasound surgery is a noninvasive treatment method that has been used to effectively treat uterine leiomyomata, but until recently has not been routinely used to treat adenomyosis. This review summarizes the current understanding of adenomyosis and high-intensity focused ultrasound, and the recent research into the safety and efficacy of this technique.
\end{abstract}

Keywords: adenomyosis, efficacy, safety, pregnancy, MRgFUS

\section{Introduction}

Adenomyosis is a benign gynecological disorder predominantly affecting premenopausal women. It is defined as the ectopic presence of endometrial glands and stroma within the uterine myometrium, with resultant reactive smooth muscle hypertrophy. ${ }^{1,2}$ Several pathological patterns exist. In some cases, there may be minimal hypertrophy of the uterus with microscopically detectable small infiltrates, confirmed only following hysterectomy. Alternatively, there may be focal lesions that may clinically resemble leiomyomata (uterine fibroids); these macroscopically visible nodules are termed adenomyomata (focal adenomyosis). It can also present as an extensive diffuse infiltration of the myometrium, termed diffuse adenomyosis. ${ }^{3}$

Although the etiology and pathophysiology of adenomyosis are not well understood, there are several hypotheses that have been suggested. ${ }^{4}$ The presence of a more invasive endometrium has been exhibited; when grown on a plain collagen matrix, stromal cells from adenomyosis have shown greater invasiveness compared with normal stromal cells. ${ }^{5}$

Differences have also been observed in smooth muscle cells from adenomyosis, where myocytes exhibit cellular hypertrophy. Moreover, it is also noted that local hyperestrogenism and steroid antagonist may induce adenomyosis. ${ }^{6} \mathrm{~A}$ marked increase of microvessel density has also been reported, which may have a role in the initiation of angiogenesis in adenomyosis. ${ }^{7}$

\section{Epidemiology}

The mean epidemiological prevalence of adenomyosis is $20 \%-30 \% .^{8}$ A recent prospective study of ultrasound (US) findings in a population of women attending general 
gynecology clinic found an overall prevalence of adenomyosis of $20.9 \%$, with a peak prevalence of $32 \%$ in women aged 40-49 years old. The prevalence varied with ethnicity, ranging from $18 \%$ to $31 \%$, with the highest prevalence in women of Asian origin, and lowest among those who identified themselves as mixed/other origin. It was noted that women with a past or current history of endometriosis were more likely to have adenomyosis, ${ }^{9}$ and retrospective data found a prevalence of $21.8 \%$ in women undergoing surgery for endometriosis. ${ }^{10}$

\section{Symptoms}

Symptoms of adenomyosis may include periodic dysmenorrhea, menorrhagia, subfertility, and pelvic pressure effects such as urinary frequency. The overlap of these symptoms with other gynecological conditions such as endometriosis or leiomyomata, coupled with the difficulty in discriminating between focal adenomyosis and leiomyomata on routine US, can create diagnostic and management difficulties for the clinician. ${ }^{3}$

\section{Current treatment options}

As a result of the often diffuse nature of adenomyosis, therapeutic options have traditionally been limited to medical management of the resulting symptoms, or definitive management by hysterectomy. As the mean age of women conceiving for the first time has increased, ${ }^{11}$ many more women will find hysterectomy to be a less acceptable option. In addition, many women have strong negative feelings toward the idea of hysterectomy. Losing their uterus can cause significant psychosocial effects, ${ }^{12,13}$ in particular concerns regarding the association of a perceived loss of femininity. ${ }^{14}$

The option of surgical excision may be considered for those who wish to preserve their uterus, but the procedure is more challenging than for myomectomy. ${ }^{15}$ If there is a defined adenomyoma lesion (focal adenomyosis), surgical excision may be possible; however, in cases of diffuse adenomyosis, debulking surgery may leave significant adenomyosis remaining in the uterus. Unlike leiomyomata, due to the ill-defined boundaries between the endometrium and myometrium, complete removal of the adenomyoma may not be possible and recurrence is more likely. ${ }^{16}$ Moreover, surgical removal can cause significant weakening of the myometrium, in particular a reduction in tensile strength, which can increase the risk of uterine rupture in a subsequent pregnancy. ${ }^{16}$

Different uterine-sparing surgical techniques, such as complete excision of adenomyosis vs cytoreductive surgery or partial adenomyomectomy, have been found to improve outcomes of dysmenorrhea, menorrhagia, and pregnancy rates.
Currently, there is no strong evidence to suggest that any one technique is superior. Given that current data regarding surgical technique are suboptimal, more studies are needed. ${ }^{17}$ When uterine-sparing surgery is performed on women with previous in vitro fertilization failures, with the aim of improving success in future treatment cycles, it is noted that a clear benefit is shown for women who are $\leq 39$ years old. ${ }^{18}$

Due to the estrogen-dependent nature of adenomyosis, symptoms can be successfully alleviated with hormonal treatments directed at disrupting the hypothalamic-pituitarygonadal axis. The most effective of these are progestagenreleasing intrauterine systems and gonadotropin-releasing hormone analogs. Combined oral contraceptives, oral progestins, danazol, and progesterone receptor modulators are also effective. ${ }^{19}$ Hormonal medications may be associated with side effects of varying tolerability, and as the underlying mechanism is lesion suppression rather than destruction, symptom relief is usually restricted to the duration of treatment. ${ }^{20-22} \mathrm{~A}$ particular difficulty of intrauterine devices is involuntary expulsion of the device, which is exacerbated by menorrhagia and an enlarged uterine cavity. However, successful symptom relief has been shown in over two-thirds of cases even in those with a uterine size $>12$ weeks, although over a third of cases experienced at least one expulsion. ${ }^{23}$

Uterine artery embolization can be used as a minimally invasive method of adenomyotic tissue destruction, which may have a more permanent therapeutic effect, but this procedure holds specific risks and the impact on future fertility and pregnancy remains uncertain. ${ }^{24}$

It can be seen, therefore, that there is currently no ideal treatment for adenomyosis, and new options are needed. Drawing on experience of treatment of leiomyomata, highintensity focused ultrasound (HIFU) has been explored as a potential new conservative treatment, and this review presents the recent advances in this field.

\section{HIFU ablation}

High-intensity ultrasound energy can be focused to a small point to produce a rise in tissue temperature sufficient to cause irreparable cell damage in the target at depth within the body. ${ }^{25}$ Diagnostic medical US commonly uses US frequencies in the range $2-15 \mathrm{MHz}$; in magnetic resonanceguided focused ultrasound surgery (MRgFUS), frequencies of between 0.9 and $1.15 \mathrm{MHz}$ are used. During diagnostic US, the temperature rise is negligible due to the US waves being distributed over a wide area, but if the waves meet at a single point, a localized high temperature at this focal point can be achieved. It is understood that coagulative necrosis is 
achieved when tissue within a focal zone is heated to a threshold temperature of $55^{\circ} \mathrm{C}-85^{\circ} \mathrm{C} .{ }^{1}$ However, $70^{\circ} \mathrm{C}-80^{\circ} \mathrm{C}$ is an optimal target temperature to ensure real tissue necrosis. ${ }^{1}$

While its potential to treat soft tissue tumors in the body was recognized in the middle of the 20th century, HIFU ablation has only recently become a widespread treatment modality. Previously, a lack of robust methods of monitoring and controlling in real time the thermal tissue damage, and its imprecision had led to markedly variable outcomes and to concerns over safety. ${ }^{3}$ In the past 10 years, HIFU has been used to treat malignant solid tumors in the liver, breast, pancreas, and bone (osteosarcoma). ${ }^{26-28}$ It has also been shown to be effective and safe for the treatment of leiomyomata. ${ }^{29}$

HIFU ablation can be performed under MR guidance, MR-guided focused US surgery, or US guidance. MR guidance has excellent anatomic resolution, very high tumor sensitivity, and high thermal imaging sensitivity. With magnetic resonance imaging (MRI), adenomyosis can be detected using T2-weighted MRI; the lesion having a junctional zone thicker than $12 \mathrm{~mm} .{ }^{30,31}$ Thus, together with the use of MRI for planning, guiding, and control of the therapy, HIFU ablation can be a safe treatment option. ${ }^{3}$

In HIFU ablation of adenomyosis, both a direct and an indirect effect are proposed to contribute to the treatment outcome. The direct effect involves protein denaturation and irreversible cell death through coagulative necrosis in the adenomyotic tissue, resulting in atrophy. The indirect effect induces necrosis or embolism of nutrient vessels, which causes obstruction to the blood supply of the adenomyotic tissue, resulting in growth inhibition. ${ }^{32}$

\section{Criteria for HIFU}

At present, there are no gold standard treatment criteria for using HIFU ablation for adenomyosis. The typical selection criteria require a patient to be 18 years and above, in premenopausal status, and to have symptomatic adenomyosis with an endometrial-myometrial junctional zone thickness of more than $3 \mathrm{~cm}$ for diffuse adenomyosis or a lesion diameter of 3-10 cm for focal adenomyosis. ${ }^{33}$

Consideration needs to be given to the position of the adenomyotic lesion to ensure that the procedure can be performed safely. It has been noted that adenomyosis in the posterior wall of the uterus can coexist with severe pelvic endometriosis, with obliteration of the posterior culde-sac and utero-rectal adhesions, characterized on MRI by a "teardrop" deformity of the rectum. ${ }^{34}$ Such cases may not be suitable for HIFU ablation due to the risk of thermal damage to the rectum. Careful review of pre-procedure MRI images is therefore required to identify extrauterine pathology and assess for increased risk of complications.

\section{Preparation for HIFU}

Protocols for patient preparation vary. Some centers use minimal additional measures, while others instigate bowel preparation in the days leading up to the procedure. To avoid bowel injury during treatment and minimize interference with treatment images, bowel loops in the acoustic pathway are pushed away or compressed by placing a degassed water balloon over the abdominal wall of the patient, or a urinary catheter is inserted. ${ }^{33,35,36}$ Skin preparation including degassing and degreasing are performed immediately before treatment to reduce the risk of skin burns. ${ }^{35}$ Some centers use sedation with fentanyl, midazolam, and/or propofol for the procedure.

\section{Studies}

A small number of studies have demonstrated the potential for effective ablation of adenomyosis with HIFU. Early studies examined the safety and efficacy of this technique. ${ }^{36-40}$ More recently, the focus has moved to examine the level of reduction of symptoms in treated patients. ${ }^{33,35,41}$ Treatment adjuncts have also been explored in an attempt to further enhance clinical effectiveness and improve efficiency. ${ }^{42}$ As the therapeutic technique is the same for both MR-guided and US-guided HIFU, studies using both guidance methods have been included in this review.

\section{Safety/complications}

Complications observed have been predominantly of Society of Interventional Radiology class A (no medical intervention required), with a small number of Society of Interventional Radiology class B (nominal therapy, no consequence). Class A adverse effects have included abdominal pain, mild sciatic/ buttock pain, genital bleeding/increased discharge, and a prolonged next period. ${ }^{37}$

Zhang et $\mathrm{al}^{33}$ reported a slightly increased frequency of abdominal pain (64\% vs $71 \%$ ) and skin reaction (0.8/2.4\%) in patients with diffuse adenomyosis compared with those with focal disease.

In their recent large analysis of 346 cases, Lee et $\mathrm{al}^{41}$ reported a range of more unusual complications, including foot drop (one case), transient unilateral leg weakness (one case), tumor lysis syndrome with transient acute prerenal failure (one case), sleep apnea due to a sedative agent (one case), first degree skin burn (five cases), second degree skin burn (three cases), and transient hematuria (ten cases). ${ }^{41}$ There were no permanent sequelae. 
It has been observed that old surgical scars can predispose a patient to skin burns over the area of the old scar when using HIFU. ${ }^{35}$ A novel scar tape has been developed that appears to reduce this risk. ${ }^{43}$

\section{Efficacy}

The principle of treatment with HIFU is that reduction in the volume of adenomyotic tissue is presumed to result in alleviation of symptoms, specifically of menorrhagia and dysmenorrhea. Treatment and follow-up protocols generally therefore include imaging to assess the overall uterine volume and volume of adenomyosis pre- and posttreatment, and the nonperfused volume of adenomyosis posttreatment. ${ }^{36,41,42}$ It is clinically important to correlate this with actual symptom resolution. In order to quantify changes in symptoms, study groups have used recognized scoring systems such as Symptom Severity Score, Uterine Fibroid Symptom and Quality of Life, and Visual Analog Scale for pain. ${ }^{35,41,44}$

\section{Reduction of tissue}

Indicators of treatment efficacy include the nonperfused volume ratio (NPVR) immediately posttreatment, and the change in overall uterine volume on later imaging. Objective treatment success is demonstrated by a significant reduction in the uterine volume in several studies, with values from $12.7 \%$ to $54 \%$. $^{36,37,41}$ The NPVR, where reported, ranges from $62.5 \%$ to $80.7 \% \%^{33,35,36,39,41}$ (Table 1 ).

\section{Reduction of symptoms}

A direct comparison is difficult due to the variation in symptom scales used in individual studies and duration of follow-up, however, all report a significant reduction in both menorrhagia and dysmenorrhea over the 3-36 months posttreatment. Those using the Uterine Fibroid Symptom and Quality of Life report an increase in QOL. ${ }^{41}$ Reported symptom reduction ranges from $25 \%$ to $83 \%$. $^{36-38,41}$ Overall clinical effectiveness (defined as any degree of improvement in symptoms) ranges from $79 \%$ to $89 \%$, with a significant proportion experiencing complete symptom relief $(39 \%-46 \%)^{33,35,38-40,42}$ These effects appear to be sustained for at least $1-2$ years $^{35,41}$ (Table 1).

Two studies have looked at the difference in efficacy between treatment of diffuse and focal adenomyosis. One study demonstrated that the rate of complete relief of dysmenorrhea at 3 months posttreatment was higher in those patients with focal adenomyosis, however, by 12 months no difference was found. There were no other significant differences in efficacy. ${ }^{33}$
Another found that the reduction in pain score was greater for focal adenomyosis at 6-month follow-up. ${ }^{38}$

\section{Treatment adjuncts}

A recent randomized controlled trial has looked at the use of oxytocin as an adjunct to treatment with HIFU. ${ }^{42}$ Research has shown that oxytocin receptors are present on the surface of nonpregnant uterine myocytes, and therefore it was hypothesized that an oxytocin infusion during HIFU treatment would cause contraction of the muscle and thus a reduction in blood flow. ${ }^{45}$ This in turn could increase the efficiency of treatment due to improved acoustic energy deposition at the target area. The trial used an infusion at a rate of $0.32 \mathrm{U} / \mathrm{min}$ oxytocin in normal saline, with a control arm of saline only.

The results were significant, with the energy efficiency factor (the amount of US energy required for the ablation of $1 \mathrm{~mm}^{3}$ of lesion) and the sonication time (time for ablating $1 \mathrm{~cm}^{3}$ of lesion) almost halved in the oxytocin group. The energy efficiency factor was reduced from $15.7 \pm 19.5$ in the control group to $8.1 \pm 9.8$ in the oxytocin group, $P=0.023$. The average sonication time was reduced from $58.1 \pm 72.7$ seconds in the control group to $30.0 \pm 36.0 \mathrm{~s}$ in the oxytocin group, $P=0.019$.

The total treatment time and energy used were similar in both groups, but the resultant NPVR was larger in the oxytocin group ( $80 \%$ vs $70 \%$ ). However, there was no significant difference in symptom relief at 12-month follow-up between the two groups. There were no observed adverse effects from the oxytocin infusion.

\section{Pregnancy after HIFU}

There have now been seven ongoing pregnancies reported after HIFU for adenomyosis, with four deliveries of healthy babies thus far. Three pregnancies had no complications and term vaginal deliveries. ${ }^{38,41,46}$ One delivery occurred by cesarean section at 32 weeks due to hemorrhage secondary to a major placenta previa. ${ }^{39}$ One study has also reported two miscarriages. ${ }^{41}$

\section{Discussion}

There is currently a lack of effective treatment options for adenomyosis, however, the disease can cause significantly distressing and debilitating symptoms particularly affecting women around the time of menstruation. In comparison with leiomyomata and endometriosis, adenomyosis has received little research attention and the disease process is poorly understood. Unlike myomectomy for leiomyomata, surgical excision of adenomyosis can be technically challenging and may have suboptimal results, as well as implications for 


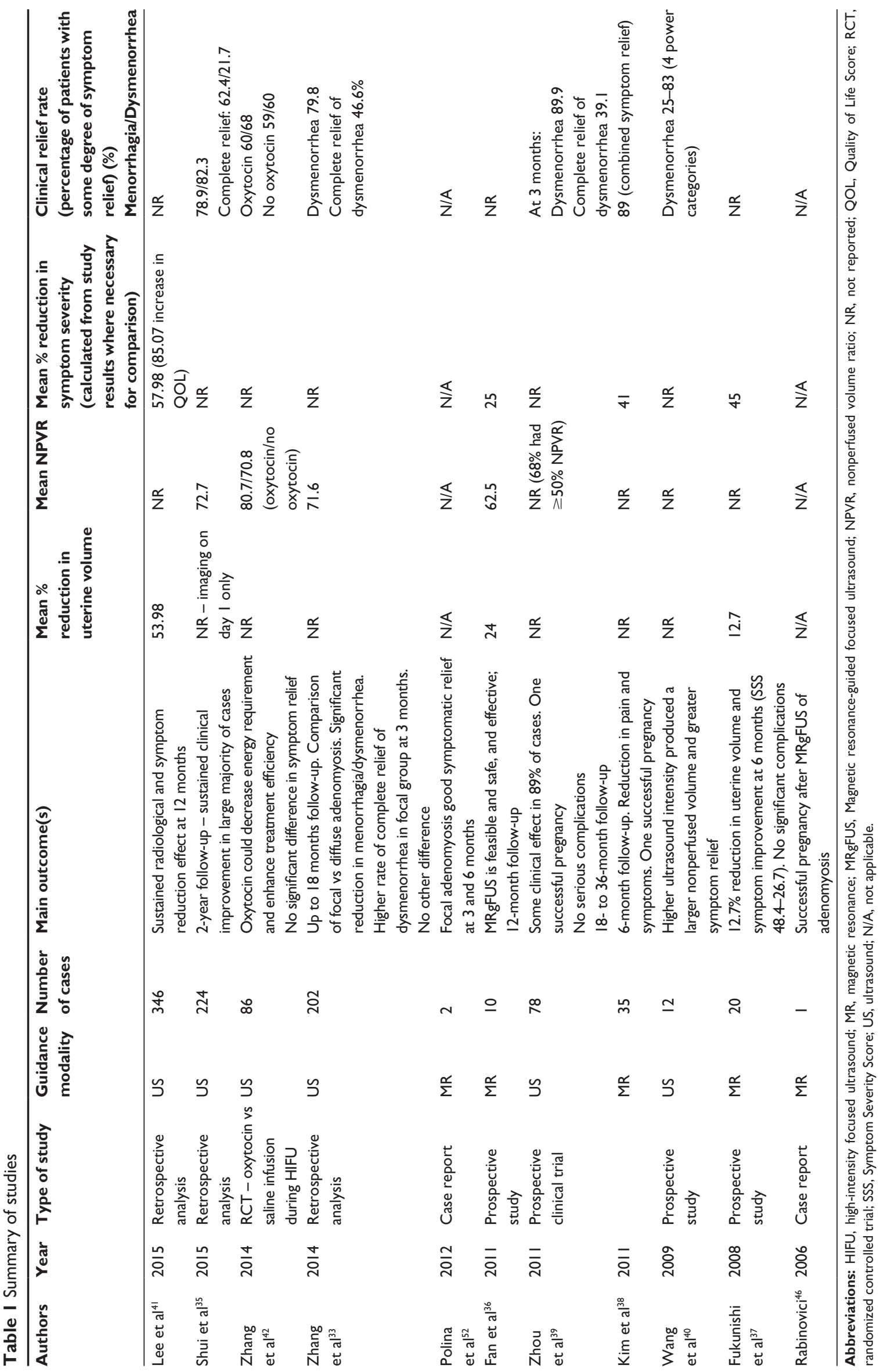


future pregnancy. ${ }^{16}$ This has traditionally left women with options only of symptom management or disease suppression with hormonal agents, or hysterectomy.

MRgFUS has been shown to be cost-effective for the treatment of uterine fibroids. ${ }^{47}$ To date, there have been no studies to assess cost-effectiveness for the treatment of adenomyosis. This is of importance, if MRgFUS is to be used routinely outside a research setting. The use of treatment adjuncts may be beneficial in this regard as increased efficiency of the procedure could in turn reduce costs, which would make the procedure more competitive when compared with uterine artery embolization (UAE) or hysterectomy. Current candidates for use as adjuncts include oxytocin infusion during the procedure as previously discussed, or preprocedure preparation with a course of gonadotropin-releasing hormone agonists, as has been shown to be effective in enhancing the effect of MRgFUS for leiomyomata. ${ }^{48,49}$

UAE has been used with some success in the treatment of leiomyomata, and more recently has been used for the treatment of adenomyosis. Current UK National Institute for Health and Care Excellence guidance advises that UAE may be offered in the treatment of adenomyosis. ${ }^{50}$ The evidence used in development of this guidance suggested UAE was efficacious in the short to medium term in relieving symptoms. These relief rates are comparable to those seen here for MRgFUS. If there is further evidence of a more sustained improvement following MRgFUS, this may become a viable alternative. Information is also needed on the rate of reintervention due to either immediate treatment failure or later symptom recurrence, which will become clear as more patients complete a 3- to 5-year follow-up posttreatment.

There is emerging evidence of successful pregnancy following MRgFUS for adenomyosis, and a significant number of pregnancies have been reported following MRgFUS for leiomyomata. ${ }^{51}$ It is difficult to draw firm conclusions from these small numbers, but given that an increase in adverse outcomes has been observed in pregnancies following UAE, it will be important to see whether MRgFUS for adenomyosis results in similar outcomes or whether it is in fact a superior treatment in this respect.

This review has included studies of both US- and MRguided FUS. While the mode of therapy is identical, there may be advantages in the use of MR guidance such as achieving a greater accuracy of ablation. US guidance is likely to be cheaper, however, when considering the cost-effectiveness, it would be important to include, but also discriminate between, both MR and US guidance.
Adenomyosis is a cause of significant morbidity and further work is needed to fully understand the disease and find effective therapies. Recent studies are encouraging, and work on uterine leiomyomata can be usefully translated to further explore treatment options. MRgFUS has been shown to be effective in relieving at least in part the symptoms of adenomyosis. For women who wish to preserve their fertility, it may prove to be a good option. However, due to the very limited numbers of reported pregnancies at present, further evidence is required to reassure clinicians of the safety of the technique before it can be recommended as a fertilitysparing intervention. For women who would be objectively suitable to undergo hysterectomy as an alternative, the cost-effectiveness of the treatment is of more importance and may prove to be a barrier to the general availability of the treatment. Improving the efficiency of the treatment with adjuncts such as oxytocin may be key in making the cost more acceptable and therefore promoting choice for all patients. Further studies, ideally in the form of randomized controlled trials, are needed to compare the effectiveness of MRgFUS with other uterine-sparing therapies such as UAE, and to explore other adjuncts such as gonadotropin-releasing hormone agonists.

\section{Disclosure}

JR Walbridge and W Chin are joint first authors in this paper. The authors report no conflicts of interest in this work.

\section{References}

1. Coakley FV, Foster BR, Farsad K, et al. Pelvic applications of MRguided high intensity focused ultrasound. Abdom Imaging. 2013;38(5): $1120-1129$.

2. Vercellini P, Viganò P, Somigliana E, Daguati R, Abbiati A, Fedele L. Adenomyosis: epidemiological factors. Best Pract Res Clin Obstet Gynaecol. 2006;20(4):465-477.

3. Rabinovici J, Stewart EA. New interventional techniques for adenomyosis. Best Pract Res Clin Obstet Gynaecol. 2006;20(4):617-636.

4. Benagiano G, Habiba M, Brosens I. The pathophysiology of uterine adenomyosis: an update. Fertil Steril. 2012;98(3):572-579.

5. Benagiano G, Brosens I. The endometrium in adenomyosis. Womens Health (Lond Engl). 2012;8(3):301-312.

6. Mehasseb MK, Taylor AH, Pringle JH, Bell SC, Habiba M. Enhanced invasion of stromal cells from adenomyosis in a three-dimensional coculture model is augmented by the presence of myocytes from affected uteri. Fertil Steril. 2010;94(7):2547-2551.

7. Kang S, Li SZ, Wang N, et al. Association between genetic polymorphisms in fibroblast growth factor (FGF)1 and FGF2 and risk of endometriosis and adenomyosis in Chinese women. Hum Reprod. 2010; 25(7):1806-1811.

8. Cockerham AZ. Adenomyosis: a challenge in clinical gynecology. J Midwifery Womens Health. 2012;57(3):212-220.

9. Naftalin J, Hoo W, Pateman K, Mavrelos D, Holland T, Jurkovic D. How common is adenomyosis? A prospective study of prevalence using transvaginal ultrasound in a gynaecology clinic. Hum Reprod. 2012;27(12):3432-3439. 
10. Di Donato N, Montanari G, Benfenati A, et al. Prevalence of adenomyosis in women undergoing surgery for endometriosis. Eur J Obstet Gynecol Reprod Biol. 2014;181:289-293.

11. Reproductive Endocrinology and Infertility Committee; Family Physicians Advisory Committee; Maternal-Fetal Medicine Committee; Executive and Council of the Society of Obstetricians, Liu K, Case A. Advanced reproductive age and fertility. J Obstet Gynaecol Can. 2011; 33(11):1165-1175.

12. Williams RD, Clark AJ. A qualitative study of women's hysterectomy experience. J Womens Health Gend Based Med. 2000;9 (Suppl 2): $\mathrm{S} 15-\mathrm{S} 25$.

13. Lewis CE, Groff JY, Herman CJ, McKeown RE, Wilcox LS. Overview of women's decision making regarding elective hysterectomy, oophorectomy, and hormone replacement therapy. J Womens Health Gend Based Med. 2000;9 (Suppl 2):S5-S14.

14. Kunde D, KhalafY. Morbidity of abdominal myomectomy: Dispelling the myth. Rev Gynaecol Pract. 2005;5:82-88.

15. Grimbizis GF, Mikos T, Zepiridis L, et al. Laparoscopic excision of uterine adenomyomas. Fertil Steril. 2008;89(4):953-961.

16. Horng $\mathrm{HC}$, Chen $\mathrm{CH}$, Chen $\mathrm{CY}$, et al. Uterine-sparing surgery for adenomyosis and/or adenomyoma. Taiwan J Obstet Gynecol. 2014;53(1): 3-7.

17. Grimbizis GF, Mikos T, Tarlatzis B. Uterus-sparing operative treatment for adenomyosis. Fertil Steril. 2014;101(2):472-487.

18. Kishi Y, Yabuta M, Taniguchi F. Who will benefit from uterussparing surgery in adenomyosis-associated subfertility? Fertil Steril. 2014;102(3):802-807.

19. Tsui KH, Lee WL, Chen CY, et al. Medical treatment for adenomyosis and/or adenomyoma. Taiwan J Obstet Gynecol. 2014;53(4):459-465.

20. Hee L, Kettner LO, Vejtorp M. Continuous use of oral contraceptives an overview of effects and side-effects. Acta Obstet Gynecol Scand. 2013;92(2):125-136.

21. Kelekci S, Kelekci KH, Yilmaz B. Effects of levonorgestrel-releasing intrauterine system and T380A intrauterine copper device on dysmenorrhea and days of bleeding in women with and without adenomyosis. Contraception. 2012;86(5):458-463.

22. Smeets AJ, Nijenhuis RJ, Boekkooi PF, Vervest HA, van Rooij WJ, Lohle PN. Long-term follow-up of uterine artery embolization for symptomatic adenomyosis. Cardiovasc Intervent Radiol. 2012;35(4) 815-819.

23. Park DS, Kim ML, Song T, et al. Clinical experiences of the levonorgestrel-releasing intrauterine system in patients with large symptomatic adenomyosis. Taiwan J Obstet Gynecol. 2015;54(4):412-415.

24. Mohan PP, Hamblin MH, Vogelzang RL. Uterine artery embolization and its effect on fertility. J Vasc Interv Radiol. 2013;24(7): 925-930.

25. Lynn JG, Zwemer RL, Chick AJ, Miller AE. A new method for the generation and use of focused ultrasound in experimental biology. J Gen Physiol. 1942;26(2):179-193.

26. Wu F, Wang ZB, Cao YD, et al. "Wide local ablation" of localized breast cancer using high intensity focused ultrasound. J Surg Oncol. 2007;96(2):130-136.

27. Chen W, Zhu H, Zhang L, et al. Primary bone malignancy: effective treatment with high-intensity focused ultrasound ablation. Radiology 2010;255(3):967-978.

28. Orsi F, Zhang L, Arnone P, et al. High-intensity focused ultrasound ablation: effective and safe therapy for solid tumors in difficult locations. AJR Am J Roentgenol. 2010;195(3):W245-W252.

29. Quinn SD, Vedelago J, Gedroyc W, Regan L. Safety and five-year reintervention following magnetic resonance-guided focused ultrasound (MRgFUS) for uterine fibroids. Eur J Obstet Gynecol Reprod Biol. 2014;182:247-251.

30. Reinhold C, Tafazoli F, Wang L. Imaging features of adenomyosis Hum Reprod Update. 1998;4(4):337-349.

31. Ascher SM, Arnold LL, Patt RH, et al. Adenomyosis: prospective comparison of MR imaging and transvaginal sonography. Radiology. 1994;190(3):803-806.
32. Dong X, Yang Z. High-intensity focused ultrasound ablation of uterine localized adenomyosis. Curr Opin Obstet Gynecol. 2010;22(4): 326-330.

33. Zhang X, Li K, Xie B, He M, He J, Zhang L. Effective ablation therapy of adenomyosis with ultrasound-guided high-intensity focused ultrasound. Int J Gynaecol Obstet. 2014;124(3):207-211.

34. Kishi Y, Suginami H, Kuramori R, Yabuta M, Suginami R, Taniguchi F Four subtypes of adenomyosis assessed by magnetic resonance imaging and their specification. Am J Obstet Gynecol. 2012;207(2):114. e1-e7.

35. Shui L, Mao S, Wu Q, et al. High-intensity focused ultrasound (HIFU) for adenomyosis: Two-year follow-up results. Ultrason Sonochem. 2015;27:677-681.

36. Fan TY, Zhang L, Chen W, et al. Feasibility of MRI-guided high intensity focused ultrasound treatment for adenomyosis. Eur J Radiol. 2012;81(11):3624-3630.

37. Fukunishi H, Funaki K, Sawada K, et al. Early results of magnetic resonance-guided focused ultrasound surgery of adenomyosis: analysis of 20 cases. J Minim Invasive Gynecol. 2008;15(5):571-579.

38. Kim KA, Yoon SW, Lee C, Seong SJ, Yoon BS, Park H. Short-term results of magnetic resonance imaging-guided focused ultrasound surgery for patients with adenomyosis: symptomatic relief and pain reduction. Fertil Steril. 2011;95(3):1152-1155.

39. Zhou M, Chen JY, Tang LD, Chen WZ, Wang ZB. Ultrasound-guided high-intensity focused ultrasound ablation for adenomyosis: the clinical experience of a single center. Fertil Steril. 2011;95(3):900-905.

40. Wang W, Wang Y, Tang J. Safety and efficacy of high intensity focused ultrasound ablation therapy for adenomyosis. Acad Radiol. 2009;16(11): $1416-1423$.

41. Lee JS, Hong GY, Park BJ, Kim TE. Ultrasound-guided high-intensity focused ultrasound treatment for uterine fibroid and adenomyosis: a single center experience from the Republic of Korea. Ultrason Sonochem. 2015;27:682-687.

42. Zhang X, Zou M, Zhang C, et al. Effects of oxytocin on high intensity focused ultrasound (HIFU) ablation of adenomysis: a prospective study. Eur J Radiol. 2014;83(9):1607-1611.

43. Yoon SW, Seong SJ, Jung SG, Lee SY, Jun HS, Lee JT. Mitigation of abdominal scars during MR-guided focused ultrasound treatment of uterine leiomyomas with the use of an energy-blocking scar patch. J Vasc Interv Radiol. 2011;22(12):1747-1750.

44. Spies JB, Coyne K, Guaou Guaou N, Boyle D, Skyrnarz-Murphy K, Gonzalves SM. The UFS-QOL, a new disease-specific symptom and health-related quality of life questionnaire for leiomyomata. Obstet Gynecol. 2002;99(2):290-300.

45. Richter ON, Kübler K, Schmolling J, et al. Oxytocin receptor gene expression of estrogen-stimulated human myometrium in extracorporeally perfused non-pregnant uteri. Mol Hum Reprod. 2004;10(5): 339-346.

46. Rabinovici J, Inbar Y, Eylon SC, Schiff E, Hananel A, Freundlich D. Pregnancy and live birth after focused ultrasound surgery for symptomatic focal adenomyosis: a case report. Hum Reprod. 2006;21(5): 1255-1259.

47. Zowall H, Cairns JA, Brewer C, Lamping DL, Gedroyc WM, Regan L. Cost-effectiveness of magnetic resonance-guided focused ultrasound surgery for treatment of uterine fibroids. BJOG. 2008;115(5):653-662.

48. Smart OC, Hindley JT, Regan L, Gedroyc WG. Gonadotrophin-releasing hormone and magnetic-resonance-guided ultrasound surgery for uterine leiomyomata. Obstet Gynecol. 2006;108(1):49-54.

49. Smart OC, Hindley JT, Regan L, Gedroyc WM. Magnetic resonance guided focused ultrasound surgery of uterine fibroids - the tissue effects of GnRH agonist pre-treatment. Eur J Radiol. 2006;59(2):163-167.

50. Uterine artery embolisation for treating adenomyosis NICE interventional procedure guidance 473. National Institute for Health and Care Excellence; 2013.

51. Bohlmann MK, Hoellen F, Hunold P, David M. High-intensity focused ultrasound ablation of uterine fibroids - potential impact on fertility and pregnancy outcome. Geburtshilfe Frauenheilkd. 2014;74(2):139-145.

52. Polina L, Nyapathy V, Mishra A, Yellamanthili H, Vallabhaneni MP. Noninvasive treatment of focal adenomyosis with MR-guided focused ultrasound in two patients. Indian J Radiol Imaging. 2012;22(2): 93-97. 
Research and Reports in Focused Ultrasound

Dovepress

\section{Publish your work in this journal}

Research and Reports in Focused Ultrasound is an international, peerreviewed, open access online journal publishing original research, study protocols, reports, editorials, reviews and commentaries on all aspects of focused ultrasound technologies for the treatment of cancer and other diseases. The manuscript management system is completely is all easy to use. Visit http://www.dovepress.com/testimonials.php to read real quotes from published authors.

Submit your manuscript here: http://www.dovepress.com/research-and-reports-in-focused-ultrasound-journal 